EXTENDED REPORT

\title{
Human preocular mucins reflect changes in surface physiology
}

\author{
M Berry, R B Ellingham, A P Corfield
}

See end of article for authors' affiliations

.....................

Correspondence to: Monica Berry PhD, Mucin Research Group, University of Bristol, Bristol Eye Hospital, Bristol BS1 2LX, UK; mon.berry@ bristol.ac.uk

Accepted for publication 11 August 2003
Br J Ophthalmol 2004;88:377-383. doi: 10.1136/bjo.2003.026583

Background/aims: Mucin function is associated with both peptide core and glycosylation characteristics. The authors assessed whether structural alterations occurring during mucin residence in the tear film reflect changes in ocular surface physiology.

Methods: Ocular surface mucus was collected from normal volunteers as $\mathrm{N}$-acetyl cysteine (NAcCys) washes or directly from the speculum after cataract surgery. To assess the influence of surface health on mucins, NAcCys washings were also obtained from patients with symptoms, but no clinical signs of dry eye (symptomatics). Mucins were extracted in guanidine hydrochloride (GuHCl) with protease inhibitors. Buoyant density of mucin species, a correlate of glycosylation density, was followed by reactivity with antipeptide core antibodies. Mucin hydrodynamic volume was assessed by gel filtration on Sepharose CL2B. Results: Surface fluid and mucus contained soluble forms of MUC1, MUC2, MUC4, and MUC5AC and also the same species requiring DIT solubilisation. Reactivity with antibodies to MUC2 and MUC5AC peaked at $1.3-1.5 \mathrm{~g} / \mathrm{ml}$ in normals, while dominated by underglycosylated forms in symptomatics. Surface mucins were predominantly smaller than intracellular species. MUC2 size distributions were different in symptomatics and normals, while those of MUC5AC were similar in these two groups.

Conclusions: A reduction in surface mucin size indicates post-secretory cleavage. Dissimilarities in surface mucin glycosylation and individual MUC size distributions in symptomatics suggest changes in preocular mucin that might precede dry eye signs.
$\mathrm{T}$ a good approximation, the mucin phenotype of a tissue is controlled by mucin genes and glycosyl transferases. The genes dictate the peptide cores to be synthesised at each developmental and physiological state. Galactosyl transferases activity, the sequential insertion of sugars onto the oligosaccharide chains, is influenced by availability and occupancy of glycosylation sites and concentration of nucleotide sugars. ${ }^{1-3}$ Mucin gene expression in the external ocular epithelia has been well documented in humans ${ }^{4-7}$ and animals. ${ }^{89}$ The presence of mature mucins on the human ocular surface and in tears has also been demonstrated. ${ }^{6}{ }^{10}{ }^{11}$ The biochemical characteristics of preocular mucins that determine their physiological roles at the ocular surface have yet to be identified. Hydrophobic regions in the peptide core could mediate aggregation or adhesion; charge-rich areas could interact in a non-specific manner while oligosaccharide epitopes might mediate specific adhesions.

We carried out a biochemical characterisation of intracellular mucins, which had not been exposed to secretory and surface agents, and of the populations present in the ocular surface environment, to assess whether there are any systematic differences between the two that might indicate the nature of mucin function in the tears.

The first step in the biochemical characterisation of human preocular mucins was to address the intracellular forms. ${ }^{12-14}$ We used these conjunctival extracted mucins to identify the mature mucin gene products, assess glycosylation, and investigate their native structure ${ }^{15}{ }^{16}$ and interactions ${ }^{17}$ as a basis for evaluating any changes at secretion or during residence in the preocular fluid. Proteolytic cleavage sites in MUCl and MUC4, ${ }^{818}$ and cleavage:C-terminal sequences from gel forming mucins MUC5AC ${ }^{19}$ and MUC2 $2^{20-22}$ suggest that these poorly understood events are part of the biosynthetic pathway leading to the production of functional glycocalyx and mucus. To specify structural modifications in ocular surface mucins, we followed mucin gene products during the progression from storage granules or subcellular membranes to the locations where physiological function is performed, as shown schematically in figure 1. Image A, built from a confocal image of normal human conjunctiva and atomic force microscope images of mucin molecules and bacteria, indicates what might happen to secreted mucin in the preocular fluid. A more detailed schematic of secreted mucin architecture is shown in figure $1 \mathrm{~B}$, to assist readers unfamiliar with this group of molecules.

Among conjunctival intracellular mucins we detected forms which maintained a very large hydrodynamic volume after reduction and alkylation while others were reduced in size, the latter indicating polymers linked by disulphide bonds. ${ }^{13}$ (The volume of the molecule in solution is the basis on which polymers are fractionated by size exclusion chromatography.) A substantial (and reversible) decrease of molecular length was observed by atomic force microscopy after the reduction of purified intracellular ocular mucins. ${ }^{16}$ Reduction with dithiothreitol breaks the disulphide bonds linking mucin subunits in the secreted mucin polymers (see fig lB). The reformation of these bonds can be blocked by alkylation. Short oligosaccharide chains ${ }^{12} 23$ and sparsely glycosylated regions tens of nanometres in length ${ }^{15}$ suggest that ocular mucins might be susceptible to cleavage during their residence in the preocular fluid. These results raise the possibility that some mucins species might be more susceptible to cleavage than others, and therefore that the distribution of hydrodynamic volumes of mucins at the ocular surface merits investigation.

The first aim of our project was to assess whether all the mucin species present in conjunctival extractions can be identified in the preocular fluid. These include the transmembranal mucins MUCl and MUC4, the main goblet cell mucin MUC5AC, and MUC2, whose distribution in the 
A
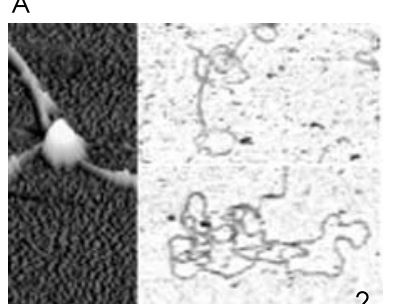

B
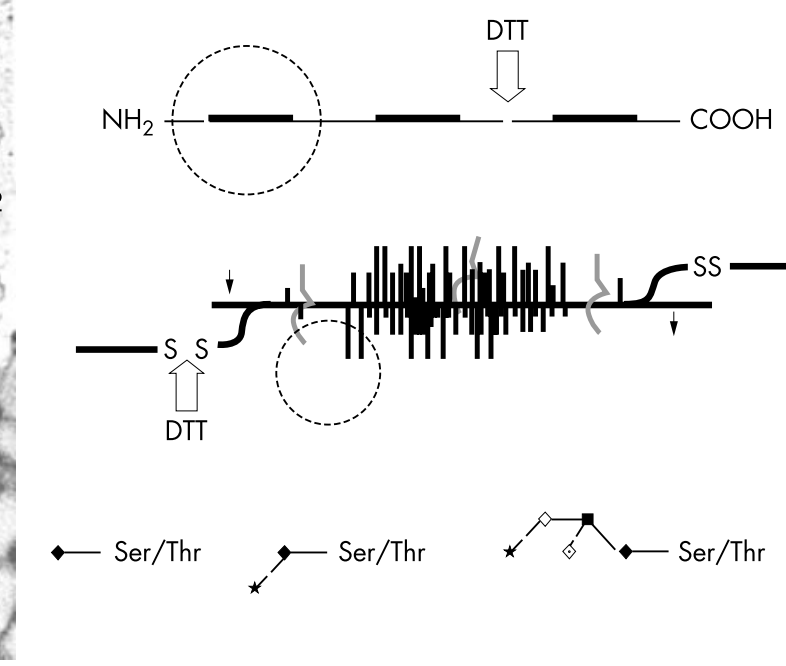

Figure 1 Mucins and tears. (A1) In the conjunctiva, secreted mucins are stored densely packed and dry in the granules of a goblet cell. On secretion mucin molecules hydrate, increasing thousands-fold in volume. (A2) In the tears, enzymatic activities, including from bacteria (top left), might cause the molecules to become smaller and less glycosylated. (The confocal image of the conjunctiva and goblet cell granules was obtained in collaboration with Julie Crewe. The atomic force microscope images of mucin polymers and bacterium (with extruded exopolysaccharide) were obtained in collaboration with Dr T J McMaster and Debra Brayshaw, HH Wills Physics Laboratory, University of Bristol.) (B) A secreted polymeric mucin is built by a linear concatenation of subunits linked by disulphide bonds. The middle section is a magnification of a subunit structure emphasising the highly O-glycosylated regions. Three common

oligosaccharides in human ocular mucins are shown in the lower sextion: Tn, SialyITn and Sialyl-Le ${ }^{x}$. Sugar symbols: $\mathrm{N}$-acetylgalactosamine, sialic acid, $*$; fucose, $\diamond$; galactose, $\diamond$; $\mathrm{N}$-acetylgluctosamine, $\mathbf{a}$. Arrows indicate the sites of DTT action; arrowheads the potential proteolytic sites cleaved at or after secretion. conjunctival epithelium is still unresolved. MUC16, now known to be one of the membrane bound mucins of the ocular surface ${ }^{24}$ was not studied.

The second aim was to ascertain which changes, if any, occur during mucin residence on the ocular surface. For this reason normal ocular surface was sampled in two ways: directly, after a short period (10-15 minutes) of accumulation under irrigation during cataract surgery, and after solubilisation by $\mathrm{N}$-acetyl cysteine (NAcCys). The first method was considered akin to extraction from tissue, the second as a direct comparison with patient samples. Preocular mucins were collected from patients with symptoms but no signs of dry eye disease, a group termed symptomatics. These were included to assess whether structural alterations in preocular mucins are sensitive to the quality of the ocular surface.

A comparison between normal surface derived mucins and analogous conjunctiva extracted forms needed to be carried out to enable the detection of pathological changes through non-invasive sampling of the ocular surface.

\section{METHODS}

\section{Collection of samples}

Following the tenets of the Declaration of Helsinki, with informed consent from all subjects and with approval from the committees for ethics in research, ocular surface mucins were obtained from patients and normal volunteers either as $\mathrm{N}$-acetyl cysteine (NAcCys) washings or directly, without chemical alteration, from specula at the end of cataract surgery. NAcCys is a routinely used mucolytic in dry eye syndromes because it solubilises mucins by cleaving disulphide bonds.

A drop $(20 \mu \mathrm{l})$ of preservative free $5 \% \mathrm{~N}$-acetyl cysteine (Moorfields Hospital, London, UK) was instilled onto the ocular surface and allowed to dwell for 1 minute while the donor pulled puncta medially out of the lacrimal pool. The accumulated fluid was collected with a sterile plastic pipette (Micropastette, Alpha Laboratories, Eastleigh, UK) and the ocular surface rinsed twice or three times with successive $20 \mu \mathrm{l}$ aliquots of sterile saline (Minims, Chauvin Pharmaceuticals, Romford, UK) to collect all the dissolved mucus. Each surface fluid sample was immediately mixed with excess $4 \mathrm{M} \mathrm{GuHCl}$ containing protease inhibitors, ${ }^{12}$ and kept at $4^{\circ} \mathrm{C}$. This method was used to obtain normal NAcCys mucus (repeated sampling of two normal volunteers). NAcCys mucus was obtained with the same method from 42 patients attending a general outpatient clinic at the Bristol Eye Hospital (female/male ratio 8:5). These patients presented with dry eye symptoms (itchiness, dryness) but no clinical dry eye signs (normal tear break up time and Schirmer tests) and therefore could not be assigned to a diagnostic category according to current dry eye diagnostic classifications. ${ }^{25-27}$

Twelve patients free of ocular surface pathology and who did not complain of any dry eye symptoms (female/male ratio 2:1) allowed us to collect surface mucus accumulated during cataract surgery. All eyes were irrigated during surgery, according to routine procedures. After speculum removal from the eye, at the end of surgery, the mucus was wiped with dry microsponges that were immediately thereafter inserted into $\mathrm{GuHCl}$ and protease inhibitors, as above.

\section{Extraction and purification of mucins}

Conjunctival fragments were extracted in $4 \mathrm{M} \mathrm{GuHCl}$ with protease inhibitors as previously described. ${ }^{12}$ All eyes are collected within 24 hours and processed within 48 hours of death, with continuous $4^{\circ} \mathrm{C}$ storage. We have previously shown that this extraction followed by a purification method identical to that followed here for surface samples produces very stable patterns of mucins, irrespective of the donor's age and sex. ${ }^{12}$ 
For mucus collected without chemical modification, supernatant was removed from the pooled microsponges, which were centrifuged to dryness. A fresh portion of $\mathrm{GuHCl}$ containing $10 \mathrm{mM}$ DDT was then added, to obtain a further, DTT, extraction. These, as well as the NAcCys collections, were each mixed with caesium chloride ( $\mathrm{CsCl}$, Sigma, Poole, UK) to a starting density of $1.4 \mathrm{~g} / \mathrm{ml}$ (final volume $13 \mathrm{ml}$ ) and centrifuged at $100000 \mathrm{~g}$ for 24 hours at $10^{\circ} \mathrm{C}$ in a Beckman preparative ultracentrifuge with a $70.1 \mathrm{Ti}$ rotor (Beckman Instruments, Palo Alto, CA, USA). Aliquots of $0.5 \mathrm{ml}$ were collected, starting at the top of the gradient, and their density established by weighing. Banding in a $\mathrm{CsCl}$ density gradient, the buoyant density of the sample, is dictated by the density of glycosylation, irrespective of molecular size. In such a gradient lipids, peptides, and proteins segregate from mucins, as do nucleic acids. ${ }^{28}$

Hydrodynamic volume distributions were obtained by size exclusion chromatography, in PBS, on Sepharose CL2B (Pharmacia Biotech, Uppsala, Sweden), as previously described. ${ }^{12}$ Previous experiments indicated that PBS and $\mathrm{GuHCl}$ yield very similar elution profiles, suggesting no interactions between mucins and the Sepharose matrix.

\section{Mucin identification}

The presence of mucins was established by reactivity, on Immobilon (Immobilon P, Millipore, Bedford, MA, USA), with well characterised antibodies to peptide core domains within and outside the tandem repeat region of mucins ${ }^{29-32}$ (table 1). Our previous publications demonstrate that the antipeptide core antibodies reliably recognise discrete mucins present in the ocular tissue and in tears. ${ }^{10}{ }^{12}{ }^{14}$ Reactivity with ocular mucins has also been established on western blots after $1 \%$ agarose gel electrophoresis of purified intracellular and surface mucins. Molucella laevis lectin (MLL) ${ }^{33}{ }^{34}$ and antibody TKH2 against Sialyl Tn were used to assess the distribution of Tn and Sialyl Tn epitopes.

Conjunctiva extracted material was reduced and alkylated on Immobilon ${ }^{35}$ to assess differences between antibody binding to molecules extracted after disulphide bond cleavage. The exhaustive washes in PBS entailed by the dot blotting method ensured comparable conditions for antibody or lectin binding irrespective of the origin of material.

Reactivities with different antibodies were visualised using appropriate horse radish peroxidase (HRP) tagged secondary antibodies (Sigma) and diaminobenzidine (Sigma), and their intensities assessed by image analysis using LabWorks4 (UVP, Inc, Upland, CA, USA). This automated method integrates grey intensities of equal defined areas and gives a result in arbitraty units. Lack of appropriate standards for individual ocular mucins and possible differences in antibody sensitivity to mucins with different degrees of glycosylation and/or molecular folding, render the assessment of pattern

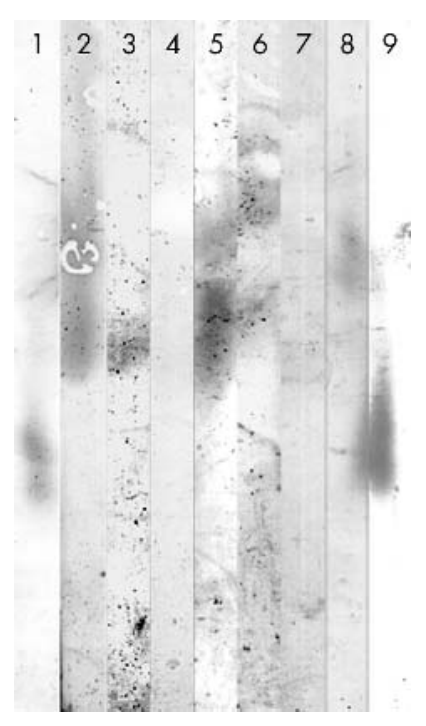

Figure 2 Reactivity with antibodies against peptide cores of ocular mucins. Ocular mucins were further purified from 1.35$1.4 \mathrm{~g} / \mathrm{ml}$ buoyant density range. Lanes 1 and 9: High range molecular weight markers, used to compare different runs. MUC2 reactivity with antibody LUM2-3 lane 2, conjunctival intracellular mucin; lane 3, ocular surface mucin; lane 4, secondary antibody only control. MUC5AC reactivity with antibody $45 \mathrm{M} 1$ to MUC5AC C-terminal sequence: lane 5 , conjunctival intracellular mucin; lane 6, ocular surface mucin. MUC4 reactivity with antibody 4F12; lane 7 , conjunctival intracellular mucin; lane 8 , ocular surface mucin.

more informative than that of reactivity magnitude. Therefore, after background subtraction, all results are presented on the same scale, from minimal to maximal intensity of reactivity, to provide a specific qualitative image of the physiologically active proportion of mucins in the sample.

\section{RESULTS}

Antibody reactivity with ocular mucins

The ability of established antibodies to the peptide core of MUC5AC, MUC4, and MUC2 (table 1) to cross react with surface mucus and purified human ocular mucins has been assessed on blots of agarose electrophoresis gels (fig 2). Mucin migration into the gel, the mobility, varied depending on the site of collection; intracellular MUC2 was of less or equal mobility to its surface analogue; while surface MUC5AC and MUC4 were less mobile than the respective surface populations. Secondary antibody only control lanes showed no cross reaction, therefore only one is shown in figure 2 .

\section{Buoyant density distribution of mucin gene products in surface eye mucus}

NAcCys collections (that is, reduced before collection) did not contain any microscopic insoluble aggregates that required addition of DTT for solubilisation in guanidine. No loss of sensitivity of reactivity with antibodies occurred after reduction and alkylation on Immobilon. ${ }^{35}$

The four mucin gene products identified in intracellular mucins from conjunctival extractions-namely, MUC1,

\begin{tabular}{|c|c|c|c|c|c|}
\hline Mucin & Antibody & $M / P^{*}$ & Peptide location & $\begin{array}{l}\text { Antibodies were donated } \\
\text { by }\end{array}$ & References \\
\hline MUC5AC & $45 \mathrm{Ml}$ & $M$ & C terminal & J Bara & 29 \\
\hline MUC5AC & LUM 5-1 & $\mathrm{P}$ & C terminal to VNTR & I Carlstedt & 31 \\
\hline MUCl & $\mathrm{BC} 2$ & M & VNTR† & M McGuckin & 30 \\
\hline MUC2 & LUM2-3 & $\mathrm{P}$ & C terminal to VNTR & I Carlstedt & 31 \\
\hline MUC4 & $\begin{array}{l}\text { M4.171 and } \\
\text { M4.275 }\end{array}$ & $M$ & VNTR & M McGuckin & 38 \\
\hline MUC4 & $4 \mathrm{~F} 12$ & M & ASPG2 & $\begin{array}{l}\text { Developmental Studies } \\
\text { Hybridoma Bank, } \\
\text { lowa University, USA }\end{array}$ & \\
\hline
\end{tabular}



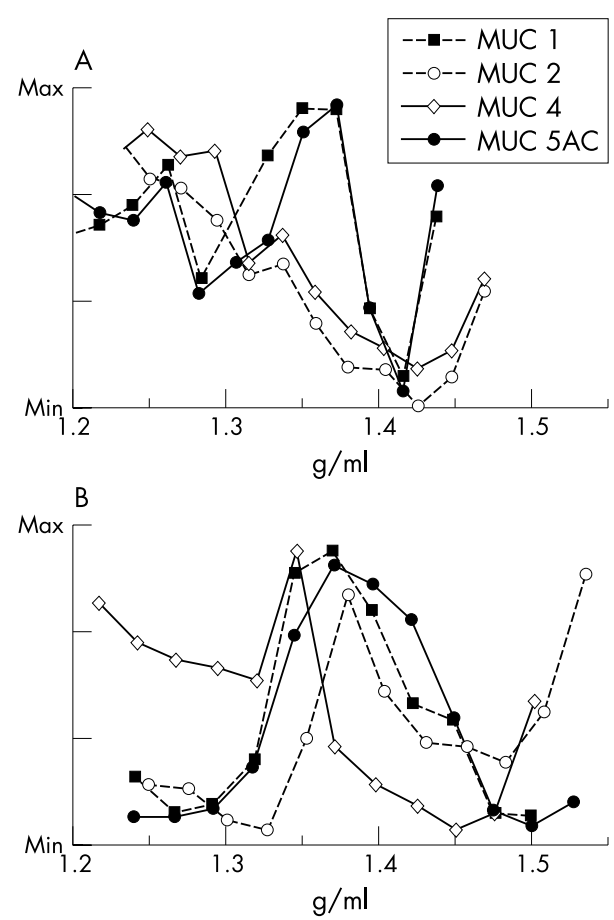

C

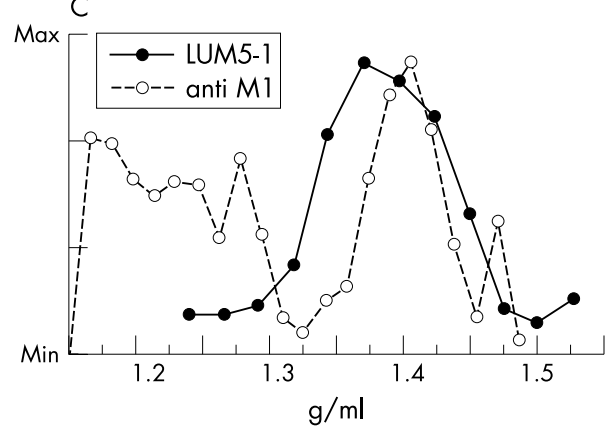

Figure 3 Distribution of mucins across the density gradient. (A) Conjunctiva extracted mucins. Proteins (peptide cores) and mucins with low density of glycosylation band at low buoyant densities on $4 M$ $\mathrm{GuHCl} / \mathrm{CsCl}$ gradients. The presence of precursors is manifest in the strong reactivity with all anti-mucin antibodies at buoyant densities below $1.3 \mathrm{~g} / \mathrm{ml}$. (B) Surface mucins from $\mathrm{N}$-acetyl cysteine washings. Mucins with mature glycosylation, which classically band between 1.3$1.5 \mathrm{~g} / \mathrm{ml}$ in these gradients, dominate the patterns of reactivity. (C) Reactivity with LUM5-1 and antiM1 to MUC5AC in N-acetyl cysteine washings from normal volunteers. Surface MUC5AC mucins do not react identically with antibodies to sequences $C$-terminal to the tandem repeat domain. In mucins extracted from conjunctival tissue the patterns obtained with these two anti-MUC5AC antibodies are very similar.

MUC2, MUC4, and MUC5AC (fig 3A) were identified in the surface mucus collected after NAcCys solubilisation from normal individuals (fig 3B). Reactivity with antibodies to mucin peptide core, except MUC4, peaked in the classic buoyant density range of mature mucins, $1.3-1.5 \mathrm{~g} / \mathrm{ml}$. Surface washings lacked mucins with low buoyant density, with the notable exception of MUC4. NAcCys collected surface MUC5AC reacted differently with the two antibodies used, antiMl and LUM5-1 (fig 3C), unlike conjunctival extractions where the two patterns overlap (not shown).

Normal surface mucus collected without chemical change (that is, directly off the speculum) yielded further mucins on extraction with DDT, suggesting that it contained microscopic insoluble aggregates. Both soluble and insoluble forms contained MUC2 and MUC5AC (fig 4). The pattern of MUC2 reactivity was similar in soluble and insoluble fractions and
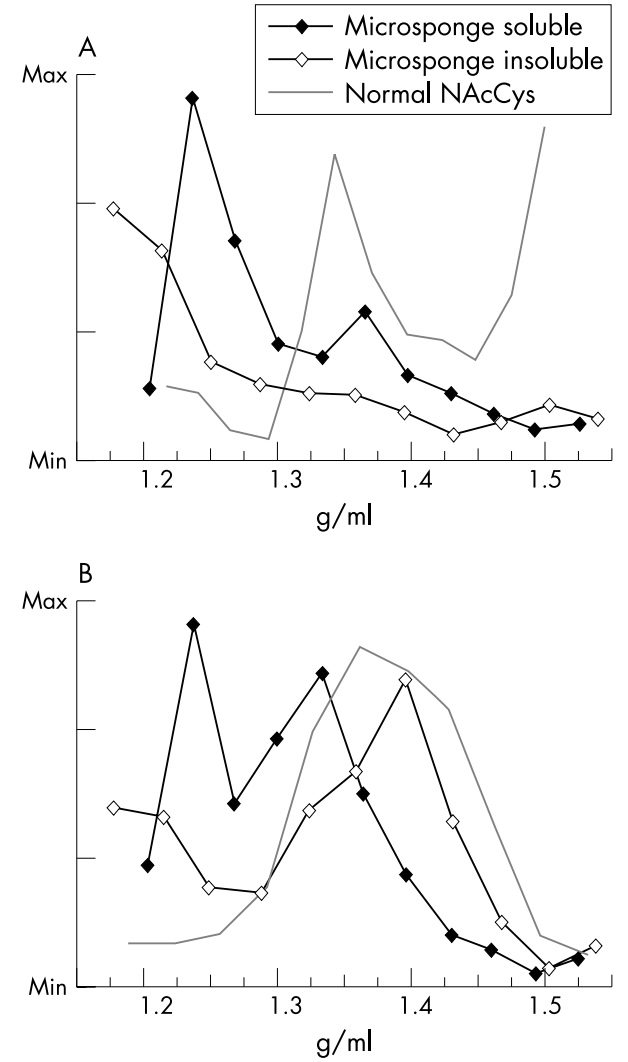

Figure 4 Soluble and insoluble mucins in direct collections of surface mucus. (A) Reactivity with LUM2-3 to MUC2 in soluble and insoluble fractions from mucus accumulating on the speculum during cataract surgery. The distribution is markedly different from that of mucins solubilised with NAcCys (solid line). (B) Patterns of LUM5-1 to MUC5AC in soluble (solid symbols) and insoluble (open symbols) fractions from speculum collections. Note the different distribution in NAcCys collections (solid line).

dominated by forms with buoyant density below $1.3 \mathrm{~g} / \mathrm{ml}$ (fig 4A). Soluble MUC5AC reactivity was shifted at buoyant densities lower than in the insoluble fraction and NAcCys collections from normal ocular surfaces (fig 4B).

Surface mucus of symptomatic patients, with symptoms but no signs of dry eye, was positive with all antibodies to the

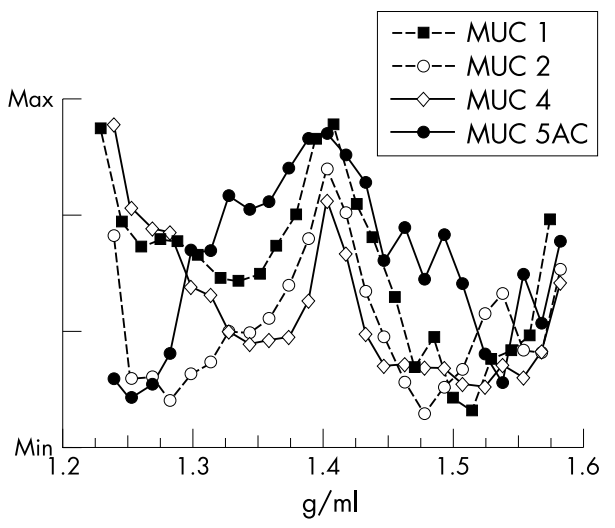

Figure 5 Mucins from symptomatic patients. Mucin banding tested with antibodies against MUC1, MUC2, MUC4, and MUC5AC in caesium chloride gradients of preocular fluid collected after NAcCys treatment. Reactivity with these antibodies covers a larger range of buoyant densities than for normal ocular surface mucins. 
four mucins tested in this study (fig 5). The reactivity extended from 1.2 to $1.6 \mathrm{~g} / \mathrm{ml}$, and peaked between 1.35 and $1.45 \mathrm{~g} / \mathrm{ml}$. Individual mucin patterns were different in normals and symptomatics. MUCl reactivity was enhanced at low buoyant densities in symptomatics. MUC4 distribution was similar in normals and symptomatics. MUC5AC had both lower and higher buoyant density than in normals. MUC2 was shifted to lower buoyant densities (fig 5).

\section{Hydrodynamic volumes of surface MUC2 and MUC5AC}

The distribution of hydrodynamic volumes of MUC2 was markedly different in normal (fig 6A) and symptomatic eyes (fig 6B): in normals the most buoyant MUC2 was present throughout the elution, while in symptomatic eyes it was confined to the terminal volume $(\mathrm{Vt})$ - that is, $<10^{5} \mathrm{Da}$. Normal surface $1.4-1.5 \mathrm{~g} / \mathrm{ml}$ MUC2 showed the strongest reactivity in the $\mathrm{Vt}$, while in symptomatics it peaked in the excluded (Vo, $>4-5 \times 10^{6} \mathrm{Da}$ ) and included (Vi) volumes.

The hydrodynamic volume of MUC5AC was similar in normal (fig 6C) and symptomatic mucus collections (fig 6D), with the exception of $1.3-1.4 \mathrm{~g} / \mathrm{ml}$ MUC5AC whose hydrodynamic volume was smaller in the latter.

Widespread TKH2 reactivity was observed in normal surface mucins, while Sialyl Tn epitopes were absent from mucins of Vo symptomatic patients. MLL cross reaction, which displays Tn and sialylTn oligosaccharides, decreased steadily with decreasing hydrodynamic volumes in mucins from symptomatic patients.
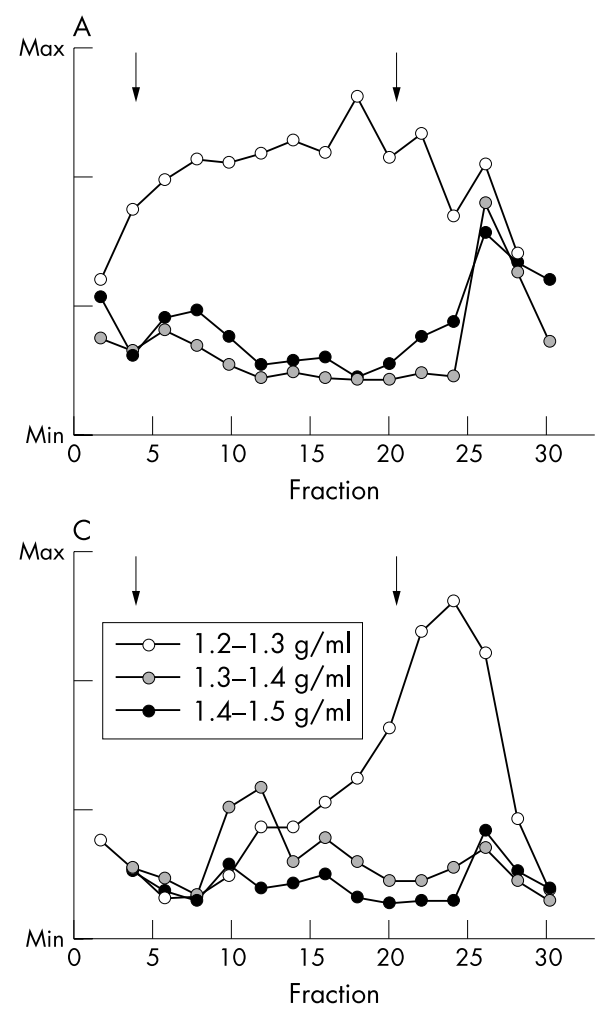

\section{DISCUSSION}

This study was undertaken to compare representative samples from each phase of mucin biology, from tissue to surface. A comparison of mucin presence and patterns in mucus from normal volunteers and patients was undertaken to establish firstly how normal surface mucins differ from pristine tissue extracted homologues and secondly to assess whether changes in the mucin make-up of the preocular fluid accompany the onset of dry eye symptoms. We did not seek to assess the quantity of mucin in each subpopulation. We used biochemical techniques validated for other mucin systems to ensure, firstly, the collection of the whole complement of mucins (hence the use of DTT and NAcCys), and, secondly, to obtain an optimal separation from other components of cytoplasm or ocular surface fluid (density gradients). Variation in the density of glycosylation is a physiological characteristic of mucins, whose relation to function has yet to be fully understood. In the context of this study a survey of mucin banding in a density gradient can be used to point to the presence of mucin fragments (for example, due to C-terminal cleavage) that change the density of glycosylation and net charge of the molecule.

All mucins identified in conjunctival tissue, gel forming and membrane bound, were found in surface mucus samples, confirming that they are part of the preocular fluid. The normal ocular surface mucus is dominated by mucins with mature glycosylation (that is, $1.3-1.5 \mathrm{~g} / \mathrm{ml}$ buoyant density fig 3B), decorated by oligosaccharides containing $\mathrm{Tn}$ and sialyl Tn epitopes and relatively small hydrodynamic volumes (fig 6A, C). A comparison with the distribution of
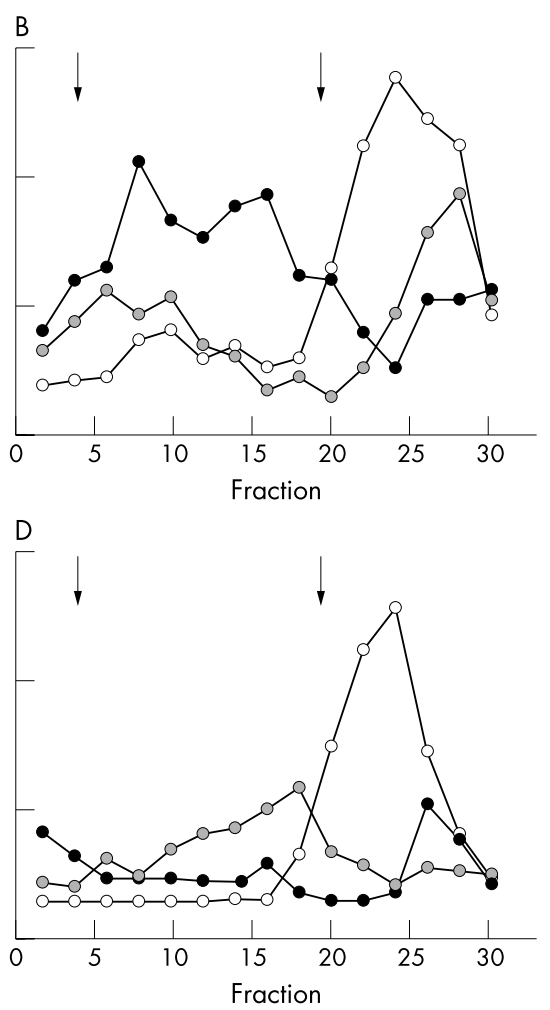

Figure 6 Hydrodynamic volume distributions of MUC2 and MUC5AC. Fractionation on Sepharose CL2B of mucins from normal volunteers (A, C) and symptomatic patients (B, D) was performed for mucins in buoyant density ranges of $1.2-1.3 \mathrm{~g} / \mathrm{ml}, 1.3-1.4 \mathrm{~g} / \mathrm{ml}$, and $1.4-1.5 \mathrm{~g} / \mathrm{ml}$. (A) Normal NAcCys surface. Mucins in the most buoyant fraction are polydisperse, while the reactivity of the MUC2 $1.3-1.5 \mathrm{~g} / \mathrm{ml}$ is restricted to the terminal volume. (B) Symptomatic eye surface. The reactivity of the most buoyant fraction is largely restricted to the terminal volume, while MUC2 1.4-1.5 g/ml reactivity peaks in the excluded and included volumes. (C) Normal NAcCys surface. Reactivity absent in Vo for MUC5AC 1.2-1.3 g/ml, but peaking in Vo for the 1.3-1.4 sample. (D) Symptomatic surface. Note shift to smaller hydrodynamic volumes for MUC5AC 1.3-1.4 g/ml. The arrows denote the start of the excluded (hydrodynamic volume too large to fractionated by Sepharose CL2B, $>4-510^{6}$ ) and terminal volumes (smaller hydrodynamic volume than resolved by Sepharose CL2B, $<10^{5}$ ), respectively. 
hydrodynamic volumes in intracellular, conjunctiva extracted, mucins ${ }^{12}{ }^{14}$ supports the notion that both stored and membrane bound mucins undergo proteolytic cleavage on or after secretion. Formation of microscopic insoluble aggregates with the participation of all mucin gene products appears to be part of the normal mucin alteration at the ocular surface, as evidenced by the DTT solubilised mucins from surface mucus accumulated during cataract surgery. It remains to be established whether these are seeded by hydrophobic entanglements of mucins in the gel, as proposed for tracheobronchial mucins. ${ }^{36}$

Surface mucins have been collected using two methods that are not entirely equivalent. Mucin synthesis is a lengthy process, therefore the increased mucin secretion during the 10-15 minutes of surgery was derived from the intracellular stores of mucin. Continuous irrigation reduced the influence of inflammatory processes on the mucus. Accumulation on the speculum comprised mucus secreted and dislodged by irrigation, and from the tarsal conjunctiva on the removal of the speculum. $\mathrm{N}$-acetyl cysteine, which reduces disulphide bridges, might emphasise changes that only occur in some subunits recovered in different subpopulations from the undivided polymer. Differences in mucin glycosylation in these samples, assayed by banding in density gradients ${ }^{35} 37$ and hydrodynamic volume indicated by gel filtration, can therefore be interpreted as evidence for mucin processing at the ocular surface. In particular, differences in reactivity of surface mucins with two antibodies to sequences in MUC5AC peptide outside the variable tandem repeat domains ${ }^{29} 31$ (fig 3C) may follow from the cleavage of the C-terminal region containing the epitope. The appearance of high buoyant density MUC2 and MUC5AC in symptomatic eyes (fig 5) suggests either preferential cleavage near a highly glycosylated region (of high negative charge) or an irreversible complexation of mucins with other surface components and decreased removal from the surface. The marked difference in the distribution of hydrodynamic volume of MUC2 in symptomatic eyes (fig 6B) compared with normals suggests that changes in surface mucins precede, or occur without, significant surface pathology. A comparison of MUC5AC (fig 6D) and MUC2 hydrodynamic volume distributions raises the possibility that mucin gene products might be affected to different extents, warranting investigation of more severe disease, within each aetiological grouping.

On a speculative note, while eyes with surface pathology are not harvested for corneal transplants, it might be interesting to consider that some of the conjunctival fragments were derived from eyes with symptoms of dry eye. Using the same techniques described here we concluded that intracellular mucins have remarkably stable distribution patterns in men and women of different ages. Differences between normal and symptomatic surface mucus imply that, in symptomatics at least, mucin alterations occur at or postsecretion and not in the conjunctival tissue itself.

In conclusion, we have shown that mucins resident in the preocular fluid encompass represent intracellular species and that, in a mucin species specific manner, they may be smaller and more sparsely glycosylated than detected within conjunctival tissue. We interpret these changes as post-secretory processing. Alterations in mucin structure reflect the condition of the ocular surface because they occur without clinically detectable (dry eye) signs.

\section{ACKNOWLEDGEMENTS}

The gift of research antibodies and lectin is gratefully acknowledged. Dr Suma Shankar is thanked for her invaluable help with patient recruitment and sample collection. This study would not have been possible without generous patient cooperation. This project was supported by grants from the Guide Dogs for the Blind (UK) and the Wellcome Trust.

\section{Authors' affiliations}

M Berry, R B Ellingham, A P Corfield, Division of Ophthalmology, University of Bristol, Bristol Eye Hospital, Lower Maudlin Street, Bristol BS1 2LX, UK

\section{REFERENCES}

1 Schachter $\mathbf{H}$, Brokhausen I. The biosynthesis of branched O-glycans. J Exp Biol 1989;43:1-22

2 Hassan $\mathrm{H}$, Reis CA, Bennett EP, et al. The lectin domain of UDP-N-acetyl-Dgalactosamine: polypeptide $\mathrm{N}$-acetylgalactosaminyltransferase-T4 directs its glycopeptide specificities. J Biol Chem 2000;275:38197-205.

3 Silverman HS, Parry S, Sutton-Smith M, et al. In vivo glycosylation of mucin tandem repeats. Glycobiology 2001;11:459-71

4 Gipson IK, Inatomi T. Mucin genes expressed by the ocular surface epithelium. Progress Ret Eye Res 1997; 16:81-98.

5 Inatomi T, SpurrMichaud S, Tisdale AS, et al. Expression of secretory mucin genes by human conjunctival epithelia. Invest Ophthalmol Vis Sci 1996;37:1684-92

6 Pflugfelder SC, Liu ZG, Munroy D, et al. Detection of sialomucin complex (MUC4) in human ocular surface epithelium and tear fluid. Invest Ophthalmol Vis Sci 2000;41:1316-26.

7 McKenzie RW, Jumblatt JE, Jumblatt MM. Quantification of MUC2 and MUC5AC transcripts in human conjunctiva. Invest Ophthalmol Vis Sci 2000;41:703-8.

8 PriceSchiavi A, Meller D, Jing $X$, et al. Sialomucin complex at the rat ocular surface: a new model for ocular surface protection. Biochem J 1998;335:457-63.

9 Tei M, Moccia R, Gipson IK. Developmental expression of mucin genes ASGP (rMuc4) and rMuc5ac by the rat ocular surface epithelium. Invest Ophthalmol Vis Sci 1999:40:1944-51.

10 Ellingham RB, Myerscough N, Gout II, et al. Soluble mucins in human aqueous tears. Biochem Soc Trans 1997;25:12S.

11 Jumblatt MM, McKenzie RW, Jumblatt JE. MUC5AC mucin is a component of the human precorneal tear film. Invest Ophthalmol Vis Sci 1999:40:43-9.

12 Berry M, Ellingham RB, Corfield AP. Polydispersity of normal human conjunctival mucins. Invest Ophthalmol Vis Sci 1996;37:2559-71.

13 Ellingham RB, Berry $M$, Stevenson $D$, et al. Secreted human conjunctival mucus contains MUC5AC glycoforms. Glycobiology 1999;9:1181-9.

14 Berry M, Ellingham RB, Corfield AP. Membrane-associated mucins in normal human conjunctiva. Invest Ophthalmol Vis Sci 2000;41:398-403.

15 McMaster TJ, Berry M, Corfield AP, et al. Atomic force microscopy of the submolecular architecture of hydrated ocular mucins. Biophys $J$ 1999;77:533-41.

16 Round A, Berry M, McMaster T, et al. Heterogeneity and persistence length in human ocular mucins. Biophys J 2003; (in press)

17 Berry M, McMaster TJ, Corfield AP, et al. Exploring the molecular adhesion of ocular mucins. Biomacromolecules 2001;2:498-503.

18 Hartman ML, Baruch A, Ron I, et al. MUC1 isoform specific monoclonal antibody $6 \mathrm{E} 6 / 2$ detects preferential expression of the novel $\mathrm{MUCl} / \mathrm{Y}$ protein in breast and ovarian cancer. Int J Cancer 1999:82:256-67.

19 Davies JR, Svitacheva N, Lannefors L, et al. Identification of MUC5B, MUC5AC, and small amounts of MUC2 mucins in cystic fibrosis airway secretions. Biochem J 1999;344:321-30.

20 Herrmann A, Davies JR, Lindell G, et al. Studies on the "insoluble" glycoprotein complex from human colon. Identification of reductioninsensitiveMUC2 oligomers and C-terminal cleavage. J Biol Chem 1999:274: 15828-36.

21 Roberton AM, Mantle M, Fahim EF, et al. The putative 'link' glycopeptide associated with mucus glycoproteins. Biochem J 1989;261:637-47.

22 Khatri IA, Forstner GG, Forstner JF. Susceptibility of the cysteine-rich Nterminal and $\mathrm{C}$-terminal ends of rat intestinal mucin muc 2 to proteolytic cleavage. Biochem J 1998:331:323-30.

23 Hicks SJ, Carrington SD, Corfield AP. Secreted canine ocular mucins have truncated oligosaccharides. Invest Ophthalmol Vis Sci 1996;37.

24 Argueso P, Spurr-Michaud S, Russo CL, et al. MUC16 mucin is expressed by the human ocular surface epithelia and carries the $\mathrm{H} 185$ carbohydrate epitope. Invest Ophthalmol Vis Sci 2003;44:2487-95.

25 Lemp MA. Report of the National Eye Institute/industry workshop on clinical trials in dry eyes. CLAO 1995;1995:221-32.

26 Bron AJ. Reflections on the tears. Eye 1997;11:583-602.

27 Pflugfelder SC, Solomon A, Stern ME. The diagnosis and management of dry eye-a twenty-five-year review. Cornea 2000;19:644-9.

28 Davies JR, Carlstedt I. In: Glycoprotein methods and protocols: the mucins, ed. Corfield AP. 3-14 Totowa, New Jersey: Humana Press, 2000

29 Bara J, Chastre E, Mahiou J, et al. Gastric M1 mucin, an early oncofetal marker of colon carcinogenesis, is encoded by the MUC5AC gene. Int J Cancer 1998;75:767-73

30 McGuckin MA, Wright G, Ward BG. Expression of a polymorphic epithelial mucin antigen defined by the monoclonal antibody bc2 in ovarian carcinoma use of the bc2 antibody for the detection of micrometastases. Am J Clin Pathol $1991 ; 96: 46-52$

31 Hovenberg HW, Davies JR, Herrmann A, et al. MUC5AC, but not MUC2, is a prominent mucin in respiratory secretions. Glycoconjugate J 1996;13:1-9.

32 Xing PX, Prenzoska J, Apostolopoulos V, et al. Monoclonal antibodies to a MUC4 peptide react with lung cancer. Int J Oncol 1997;1 1:289-95.

33 Lis $\mathrm{H}$, Sharon N. Molucella laevis lectin-an unusual protein with unique specificity. Trends Glycosci Glycotechnol 1994;6:65-74. 
34 Yonezawa S, Tachikawa T, Shin S, et al. Sialosyl-Tn antigen. Its distribution in normal human tissues and expression in adenocarcinomas. Am J Clin Path 1992;98:167-74.

35 Askoy N, Thornton DJ, Corfield A, et al. A study of the intracellular and secreted froms of the MUC2 mucin from the PC/AA intestinal cell line. Glycobiology 1999;9:739-46.
36 Bromberg LE, Barr DP Self-association of mucin. Biomacromolecules 2000;1:325-34.

37 Corfield AP, Carrington SD, Hicks SJ, et al. ocular mucins: purification, metabolism and function. Progr Ret Eye Res 1997:16:627-56.

38 Xing P-X, Prenzoska J, Apostolopoulos V, et al. In: 3rd International workshop on carcinoma-associated mucins 127. Cambridge: ICRF, 1996. 